\title{
Approaching perioperative cardiac risk: Forging new strategies against an old enemy
}

\author{
Yasmeen Golzar, MD, FACC ${ }^{a}$ \\ a Division of Cardiology, Cook County Health, Chicago, IL \\ Received May 13, 2019; accepted May 13, 2019 \\ doi: $10.1007 / \mathrm{s} 12350-019-01780-2$
}

\section{See related article, pp. $1331-1337$}

If perioperative cardiac events are the formidable enemy, then it is time to regroup the troops. Practicechanging studies have forced us to retreat from what were once common and considered to be intuitive practices, such as prophylactic preoperative revascularization or targeting sympathetic stress response with perioperative $\beta$-blockers, ${ }^{1,2}$ in favor of a more cautious and nuanced approach when risk stratifying and managing patients in the perioperative period. As the dust has settled, certain aspects of our current state are clear-perioperative myocardial infarction (MI) remains a frequently silent but dreaded complication of noncardiac surgery carrying a significant risk of 30-day mortality ${ }^{3}$; our arsenal is limited in predicting perioperative events, meager in managing them, and nearly empty in preventing them. It is time to rethink our strategy.

The most current clinical practice guidelines continue to rely on clinical risk indices and stress testing as preoperative predictors of cardiac risk. ${ }^{4}$ However, clinical risk indices, whether the well-validated RCRI or the more contemporary NSQIP MICA calculator, are known to underestimate risk of perioperative major adverse cardiac events (MACE). ${ }^{5}$ Additionally, data on the value of stress testing in predicting perioperative events are variable. While a large number of single-site studies have found that moderate to large areas of myocardial ischemia, by dobutamine stress echocardiography or stress nuclear myocardial perfusion imaging (MPI), are

\footnotetext{
Reprint requests: Yasmeen Golzar, MD, FACC, Division of Cardiology, Cook County Health, 1901 W. Harrison St, Chicago, IL 60612, USA; ygolzar@cookcountyhhs.org

J Nucl Cardiol 2020;27:1338-40.

$1071-3581 / \$ 34.00$

Copyright (C) 2019 American Society of Nuclear Cardiology.
}

predictive of perioperative MACE, none of them have shown that stress testing is able to reclassify patients to a higher or lower risk category as compared with the use of clinical risk indices alone. ${ }^{4}$ Furthermore, meta-analyses have highlighted the modest predictive value of stress nuclear MPI in predicting MACE, demonstrating that one-third of myocardial infarctions or deaths occur in patients with normal results. ${ }^{6,7}$

The variable data on the value of stress nuclear MPI in predicting events touch on the complex and multifactorial mechanism of perioperative MI. Several confluent factors including sympathetic stimulation, systemic inflammation, and wide hemodynamic fluctuations from anesthesia and surgery induce significant yet unpredictable alterations in coronary plaque morphology, making them vulnerable to myocardial supplydemand mismatch and plaque rupture. ${ }^{5,8}$ Significant ischemia on preoperative stress testing is predictive of perioperative MACE, likely identifying patients who are at risk of perioperative MI by the mechanism of demand ischemia. However, the mechanism of plaque rupture is unpredictable and can occur in non-flow limiting coronary lesions which would go undetected in preoperative stress testing, contributing to its limited negative predictive value.

Acknowledging that current risk prediction tools are suboptimal and with expanding clinical applications of advanced cardiac imaging modalities, established approaches to preoperative risk stratification warrant reexamination. In shifting the paradigm from functional testing to anatomic imaging, the authors of the VISIONCTA trial studied the prognostic value of coronary computed tomography angiography (CCTA) as a strategy for perioperative risk prediction. CCTA, with its high diagnostic accuracy in defining the degree of coronary artery stenosis and its established prognostic value in a general population, is an appealing imaging modality to consider in the preoperative setting. The results of VISION-CTA demonstrated that, compared with the RCRI alone, findings on preoperative CCTA 
improved risk estimation among patients in whom perioperative cardiac events occurred. Unfortunately, this was at the expense of CCTA overestimating risk nearly fivefold. ${ }^{9}$ Actual MACE rates were low, even among patients with severe CAD on CCTA, ${ }^{9}$ highlighting the need for further risk stratification of this population in order to avoid inappropriate further testing and possible delays in needed surgery.

In a sub-study of the VISION-CTA trial featured in this issue of the journal, Dowsley et al. ${ }^{10}$ explore whether combining functional testing and anatomic assessment of CAD could further improve prediction of perioperative MACE in patients undergoing non-cardiac surgery. The investigators studied a small subset of patients (55 out of 955) who had both CCTA and stress nuclear MPI as part of their preoperative assessment; primary outcomes were all-cause death and non-fatal MI at 30 days. They found that there was a strong correlation between the degree of obstructive CAD by CCTA and the severity of myocardial ischemia by stress MPI. Additionally, the predictive capacity of stress MPI improved when only those patients with severe CAD on CCTA were analyzed. The authors propose that CCTA could be utilized as the initial test for preoperative cardiac evaluation, and stress MPI could be used to further "refine", perioperative risk prediction in those patients with severe CAD on CCTA. ${ }^{10}$

The concept investigated in this study is novel, and the results are thought-provoking. I am unaware of any other published study employing a combination of CCTA and stress nuclear MPI for perioperative cardiac risk evaluation. However, the clinical applicability of the study findings is limited by several factors. First, the results can only be "hypothesis-generating" since the study is significantly underpowered, limiting the conclusions which can be derived from it. Second, these patients represent a high-risk cohort, both by clinical risk profile as well as actual outcomes, limiting the generalizability of these findings. Baseline characteristics of these patients are significantly different than the larger cohort of patients studied in the original VISIONCTA trial; $80 \%$ of the patients in this sub-study were men, $84 \%$ had hypertension, and $91 \%$ were smokers or ex-smokers. $53 \%$ of patients had severe CAD by CCTA and $31 \%$ had significant ischemia by MPI. 30-day MACE was $14.5 \%$ (vs $8 \%$ in the original study). ${ }^{9,10}$ Why these patients were chosen for further testing by MPI is unclear; the decision was made by individual practitioners, and one can presume it was due to concerns that these patients were high risk for perioperative MACE. Lastly, due to the paucity of women in this substudy, the relevance of these results in women cannot be ascertained.
The biggest impediment to the adoption of the authors' proposed strategy is the fact we have very little protective armor to prevent perioperative cardiac events and very few weapons at our disposal when they occur. Numerous tactics, from prophylactic medications to higher post-operative hemoglobin transfusion thresholds, have been explored but have failed to show benefit in preventing perioperative MACE. ${ }^{5}$ What is the point of improving risk prediction of perioperative cardiac events if we do not have means of preventing them? Without evidence-based preventive measures that would change outcomes, one cannot justify a strategy of stacking multiple non-invasive tests in preoperative patients, exposing them to the risks of iodinated contrast and ionizing radiation and the added cost of care, without offering any benefit.

Even if we are unable to readily adopt such a strategy into real-world practice, with their study, Dowsley et al. add to our understanding of the interplay between anatomical and functional assessment in the context of perioperative risk prediction. As we map our future steps, the advancement of hybrid non-invasive imaging modalities which provide both anatomic and functional information, such as CT-PET and CT-FFR, may hold the most promise. Moreover, being able to better identify our most vulnerable patients allows us to further investigate preventive and treatment strategies targeting this population. Since severity of myocardial ischemia has consistently been shown to be predictive of perioperative risk, ${ }^{6}$ shifting our focus to study ischemiadriven management strategies may be the most revealing. In fact, though the CARP trial did not show any benefit in preoperative revascularization of patients with angiographically defined severe stable CAD, subgroup analysis of patients with concomitant significant ischemia on preoperative stress MPI suggested that revascularization may be beneficial in this subset of patients. ${ }^{11}$ Such targeted perioperative management strategies must be revisited in large prospective trials studying our highest risk patients. Only then will we be able to forge ahead.

\section{Disclosure}

None.

\section{References}

1. McFalls EO, Ward HB, Moritz TE, et al. Coronary-artery revascularization before elective major vascular surgery. N Engl J Med 2004;351:2795-804.

2. Group PS, Devereaux PJ, Yang H, et al. Effects of extendedrelease metoprolol succinate in patients undergoing non-cardiac 
surgery (POISE trial): a randomised controlled trial. Lancet 2008;371:1839-47.

3. Botto F, Alonso-Coello P, Chan MT, et al. Myocardial injury after noncardiac surgery: a large, international, prospective cohort study establishing diagnostic criteria, characteristics, predictors, and 30day outcomes. Anesthesiology 2014;120:564-78.

4. Fleisher LA, Fleischmann KE, Auerbach AD, et al. 2014 ACC/ AHA guideline on perioperative cardiovascular evaluation and management of patients undergoing noncardiac surgery: a report of the American College of Cardiology/American Heart Association Task Force on practice guidelines. J Am Coll Cardiol 2014;64:e77-137.

5. Devereaux PJ, Sessler DI. Cardiac complications in patients undergoing major noncardiac surgery. $N$ Engl $J$ Med 2015;373:2258-69.

6. Beattie WS, Abdelnaem E, Wijeysundera DN, Buckley DN. A meta-analytic comparison of preoperative stress echocardiography and nuclear scintigraphy imaging. Anesth Analg 2006;102:8-16.

7. Kertai MD, Boersma E, Bax JJ, et al. A meta-analysis comparing the prognostic accuracy of six diagnostic tests for predicting perioperative cardiac risk in patients undergoing major vascular surgery. Heart 2003;89:1327-34.
8. Priebe HJ. Perioperative myocardial infarction-aetiology and prevention. Br J Anaesth 2005;95:3-19.

9. Sheth T, Chan M, Butler C, et al. Prognostic capabilities of coronary computed tomographic angiography before non-cardiac surgery: prospective cohort study. BMJ 2015;350:h1907.

10. Dowsley TF, Sheth T, Devereaux P, Tandon V, Chow B. Complementary preoperative risk assessment using coronary computed tomography angiography and nuclear myocardial perfusion imaging in non-cardiac surgery: a VISION-CTA substudy. J Nucl Cardiol 2019. https://doi.org/10.1007/s12350-019-01779-9.

11. Garcia S, Rider JE, Moritz TE, et al. Preoperative coronary artery revascularization and long-term outcomes following abdominal aortic vascular surgery in patients with abnormal myocardial perfusion scans: a subgroup analysis of the coronary artery revascularization prophylaxis trial. Catheter Cardiovasc Interv 2011;77:134-41.

Publisher's Note Springer Nature remains neutral with regard to jurisdictional claims in published maps and institutional affiliations. 